\title{
Magnetic resonance imaging of temporomandibular joint with anterior disk dislocation without reposition - long-term results
}

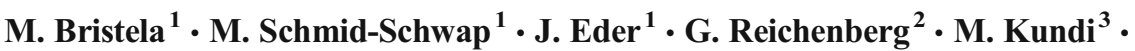 \\ E. Piehslinger ${ }^{1} \cdot$ S. Robinson ${ }^{4}$
}

Received: 26 January 2016 / Accepted: 1 March 2016/Published online: 16 April 2016

(C) The Author(s) 2016. This article is published with open access at Springerlink.com

\begin{abstract}
Objectives Anterior disk dislocation (ADD) without reposition in the temporomandibular joint (TMJ) may be associated with morphological changes in the retrodiscal region of the bilaminar zone presenting as pseudo-disk (PD). The present study was initiated to investigate the development of retrodiscal fibrosis in a period of 4-8 years and to assess if patients with a PD show differences in the clinical and radiologic findings versus patients without a PD.

Materials and Methods In a retrospective follow-up study of 33 consecutive patients with ADD without reposition in one
\end{abstract}

M. Bristela

margit.bristela@meduniwien.ac.at

M. Schmid-Schwap

martina.schmid-schwap@meduniwien.ac.at

J. Eder

jaryna.eder@meduniwien.ac.at

M. Kundi

michael.kundi@meduniwien.ac.at

E. Piehslinger

eva.piehslinger@meduniwien.ac.at

S. Robinson

s.robinson@dzu.at

1 Department of Fixed and Removable Prosthodontics, University Clinic of Dentistry, Sensengasse 2a, 1090 Vienna, Austria

2 Dental Office, Vienna, Austria

3 Institute of Environmental Health, Center for Public Health, Medical University of Vienna, Kinderspitalgasse 15, 1090 Wien, Austria

4 Diagnostic Center Urania, Laurenzerberg 2, 1010 Vienna, Austria or both TMJs, a clinical and MRI-supported evaluation was conducted 4 to 8 years after baseline diagnosis.

Results In $45 \%$ of the TMJs with ADD without reposition, a PD could be identified. Twenty-one of 31 patients who showed pain at the baseline examination (VAS mean $56 \pm 38$ ) were pain free. The mouth opening capacity (MO) of the mandible could be increased in $80 \%$. There were no statistical significant differences between patients with or without PD in these clinical features. The MRI parameters effusion and translation showed a statistical tendency for more improvement in the group with PD $(p=0.061,0.064)$.

Conclusion In about half of the patients, a structure corresponding to a pseudo-disk developed during follow-up. Pain and the mouth opening capacity improved in all patients independent of the development of a PD.

Clinical Relevance Detection of a PD during follow-up of patients with ADD without spontaneous reposition does neither predict favorable nor worse therapy response and clinical course.

Keywords MRI · TMD · Pseudo-disk · Disk position · Disk displacement without reposition

\section{Introduction}

"Temporomandibular disorder" (TMD) stands for a wide range of clinical articular and muscular problems in the orofacial region. The clinical complex of symptoms comprising articular noises, ear pain, and/or headache, frequently associated with tinnitus, dizziness, and burning tongue, was initially described by Costen and termed Costen's syndrome (Costen et al. 1934).

Clinical symptomatology can be characterized by pain, tenderness on palpation of the temporomandibular joints (TMJ) 
and masticatory muscles, articular noises, and irregular or restricted jaw function $[1,2]$. Approximately one third of the adult population shows at least one symptom of TMD [3].

TMD can be subdivided into arthrogenic and myogenic disorders [4]. Disk dislocation of the TMJ is classified as an arthrogenic disorder and categorized according to the displacement plane. Displacement of the disk in the sagittal plane is termed anterior or posterior displacement, while displacement in the transversal plane is termed medial or lateral.

Magnetic resonance imaging (MRI) of the anatomical structures has been established as internationally recognized standard for the assessment of position, morphology, and mobility of disk and condyle and evaluation of adjacent structures [3]. In this respect, three Tesla units appear superior to those with a lower field strength using the same examination time $[5,6]$.

By definition, anterior disk dislocation (ADD) is present, when the posterior border of the disk is visualized in MRI anterior to the 12 o'clock position of the condyle [7-9]. Moreover, disk position will depend on the inclination of the eminence and ventral positioning of the disk will increase with the inclination of the eminence [10]. For therapeutic management, it is relevant to assess disk mobility during mouth opening (MO). If the disk resumes its physiological position on the condyle during the jaw opening movement, this is termed ADD with reposition. However, if the articular disk remains dislocated in front of the condyle during the complete opening movement, this is termed as ADD without reposition [11]. This may result in a restriction of movement (locked joint). In $9 \%$ of temporomandibular joints with ADD with reposition, this condition will develop to ADD without reposition within a period of 3 years [12].

In MRI, the chronically displaced articular disk mostly shows a very low signal intensity and frequently a flat shape. Its posterior portion and the bilaminar zone render a lower signal than in the healthy TMJ [13]. Histologically, increased fibroblast activity, neogenesis of collagen fiber bundles, and hyalinization can be demonstrated in the TMJ with ADD without reposition [14-16]. Some authors postulate that chronic inappropriate dorsal or dorsocranial loading of the bilaminar zone can result in the formation of a pseudo-disk $[3,13,16,17]$. The histological studies of Scapino and the MRI evaluations of Katzberg show that fibrous cords from the bilaminar zone may occasionally form a "pseudo-disk".

As yet, no precise or specific data have been reported regarding the time course required for this remodeling. However, the time passed since the disk displacement may also play a major role in explaining the variability of morphologic changes.

The present study was initiated to investigate the development of retrodiscal fibrosis, a so-called pseudo-disk, in a period of 4-8 years and to assess if patients with a pseudo-disk show differences in the clinical and radiologic findings versus patients without a pseudo-disk.

\section{Material and methods}

The present study was approved by the Ethics Committee of the Medical University Vienna (EK 1742/2012). The investigations and procedures were performed after having obtained written informed consent of each participating patient.

Over the period from 2004 to 2008 , more than 2300 patients were evaluated at the outpatient unit for temporomandibular disorders of the prosthetic department of the Dental University Clinic of Vienna with part of these patients undergoing MRI with three Tesla.

Fifty-one patients meeting the inclusion criteria (ADD without reposition, having had their baseline diagnostic evaluation at least 4 years before and MRI with three Tesla at the Diagnostic Center Urania) were invited for a follow-up examination. Exclusion criteria were patient's age below 18 years, orthodontic treatment, tremor, presence of ferromagnetic objects or biostimulators, and pregnancy.

Out of the 51 patients included, 15 patients did not respond to contact by postal mail or phone and two patients refused any additional MRI. Thirty-four patients consented to participate and underwent the follow-up clinical evaluation. One of these patients did not present for the MRI.

Thus, 33 persons (66 joints) were enrolled in the study. Twenty-four joints showed no ADD without reposition, therefore, 42 joints were investigated. Twenty-eight $(85 \%)$ of the patients were female and five patients $(15 \%)$ were male. The patients had an average age of $53 \pm 18$ years (range 23-77).

At the baseline examination, collection of the patient's detailed history and evaluation of pain sensation were followed by a standardized clinical evaluation [8]. This evaluation comprises facial neurological assessment, evaluation of jaw movements, and palpation of muscles and temporomandibular joints as well as assessment of occlusion and articulation.

Pain sensation was evaluated using the Visual Analog Scale (VAS). The range between no pain and maximum pain is displayed by a color line with increasing color intensity. Using a movable bar, the patient is asked to indicate his/her pain experienced at rest, under function (e.g., chewing and mouth opening) and maximum pain experienced. On the backside of the scale, not visible for the patient, the respective numerical value $(0-100)$ is displayed.

At the baseline visit, all patients with TMJ complaints received detailed information on the function and dysfunction of the TMJ as well as about pathogenic factors and got behavioral guidelines and information about the impact of habits (e.g., nail biting, gum chewing, and clenching during the day).

Two of the patients had received no therapy at all, two patients only splint therapy, four patients physiotherapy, and 25 patients a combination of both. Splints were fabricated following the concept of frontal and canine guidance and were adapted regularly over a 
period of 3 months. Physiotherapy was conducted by experienced therapists. A therapy-package included 10 units, with session lasting $45 \mathrm{~min}$.

Prior to performance of follow-up MRI, the same clinical parameters as for the baseline examination were evaluated again.

\section{MRI scan}

Baseline and follow-up MRI were performed at the same institute to ensure equal examination conditions. Patients were positioned supine, a Sense Flex-M coil was placed exactly over the temporomandibular joint. All MRI scans were done with a three Tesla unit of Philips Intera 3.0 (Philips Medical Systems, Netherlands) with closed mouth in parasagittal and paracoronal plane and with open mouth (defined jaw opening of $30 \mathrm{~mm}$ by means of bite jig) in parasagittal plane. The slice thickness of the images was $2 \mathrm{~mm}$ (Table 1). All images were evaluated by a radiologist specialized in head and neck MRI with 27 years of professional experience.

For the assessment of morphological changes in the parasagittal plane of the joint, the central images were selected, in which the structures were best visible. The ancillary target criteria of radiologic evaluation are shown in Table 2.

\section{Statistical analysis}

In order to ensure that the prerequisite for the independence of the observations is not violated, only one joint per patient was included in the evaluation. Thus, the number of temporomandibular joints evaluated was 33 .
Only the joint showing no pseudo-disk in the baseline evaluation was included in the analysis for those patients with bilateral joint involvement. If no pseudodisk was seen in either the baseline or the follow-up evaluation, one of the two joints was randomly selected.

Statistical evaluation involved the assessment to which extent clinical and radiologic parameters in the baseline evaluation allowed for a prognosis or prediction of the development of a pseudo-disk as well as the question whether patients developing a pseudo-disk during the follow-up period could be differentiated with regard to the development of clinical and radiologic findings. The first question was assessed using logistic regression. The second question was tested using a generalized linear model with repeated measurements assuming a binomial, multinomial, or normal distribution depending on variable type. Development of a pseudodisk in clinical course was included as group factor. For all analyses, which were performed using SPSS 22.0 (IBM, USA), a $p$ value below 0.05 was taken as significance level and a $p$ value below 0.1 was considered a statistical tendency.

\section{Results}

In nine of the 33 patients included, a disk displacement without reposition could be noted in both temporomandibular joints. In 24 patients, an ADD without reposition could only be shown in one joint in the MRI. In the baseline MRI scan, two patients already showed morphological changes in the

Table 1 Magnetic resonance imaging sequences

\begin{tabular}{|c|c|c|c|c|c|c|}
\hline & \multicolumn{2}{|c|}{ T2w-TSE sequence } & \multicolumn{2}{|c|}{ T1w-TSE sequence } & \multicolumn{2}{|c|}{ PDw-TSE sequence } \\
\hline & \multicolumn{2}{|c|}{ Parasagittal closed mouth } & \multicolumn{2}{|c|}{ Paracoronal closed mouth } & \multicolumn{2}{|c|}{ Parasagittal opened mouth } \\
\hline & Baseline MRI & Follow-up MRI & Baseline MRI & Follow-up MRI & Baseline MRI & Follow-up MRI \\
\hline & 3.0 & 3.0 & 3.0 & 3.0 & 3.0 & 3.0 \\
\hline FOV & 150 & 150 & 130 & 130 & 150 & 150 \\
\hline Slices & 15 & 15 & 8 & 8 & 15 & 15 \\
\hline Slices thickness (mm) & 2 & 2 & 2 & 2 & 2 & 2 \\
\hline TSE factor & 14 & 14 & 4 & 6 & 6 & 6 \\
\hline $\mathrm{TR}(\mathrm{ms})$ & 4823 & 4147 & 550 & 551 & 2,451 & 2.375 \\
\hline $\mathrm{TE}(\mathrm{ms})$ & 80 & 80 & 13,9 & 8,4 & 20 & 20 \\
\hline Flip angle & 90 & 90 & 90 & 90 & 90 & 90 \\
\hline NSA & 4 & 2 & 3 & 4 & 4 & 2 \\
\hline Scan duration (min) & $03: 56$ & $03: 27$ & 03:39 & 04:06 & $04: 10$ & $04: 35$ \\
\hline Measured voxel size (mm) & $0.55 / 0.71 / 2.00$ & $0.55 / 0.71 / 2.00$ & $0.54 / 0.68 / 2.00$ & $0.54 / 0.68 / 2.00$ & $0.55 / 0.79 / 2.00$ & $0.55 / 0.7 / 2.00$ \\
\hline Reconstructed voxel size (mm) & $0.29 / 0.29 / 2.00$ & $0.29 / 0.29 / 2.00$ & $0.51 / 0.5 / 2.00$ & $0.51 / 0.50 / 2.00$ & $0.29 / 0.29 / 2.00$ & $0.29 / 0.29 / 2.00$ \\
\hline
\end{tabular}

FOV field of view, TSE turbo spin echo, TR time of repetition, TE echo time, NSA number of signals averaged, 
Table 2 Ancillary target criteria of radiologic evaluation

\begin{tabular}{lllll}
\hline Disk position & Anterior & Anterior-medial & Anterior-lateral & \\
\hline Disk shape & Biconcave & Flat & Biconvex & Deformed \\
Signal intensity & Homogenous & Focal hyperintensities & & Disk residues \\
Inclination of eminence & Normal & Steep & Flat & None \\
Translation & Normal & Restricted & & Disk remnants \\
Effusion & Yes & No & Sclerotic & Lateral \\
Condyle degeneration & Flattened & Cuspidated & Anterior & Retral \\
Condyle position & Distraction & Compression & \\
\hline
\end{tabular}

form of a pseudo-disk in one joint. In the follow-up scan after 4-8 years, a pseudo-disk was found in 19 of the 42 temporomandibular joints (Fig. 1a,b), while 23 joints did not show any pseudo-disk (Fig. 2a,b). The average age of the group without PD was $50 \pm 17$ years that of the group with PD $56 \pm 17$ years $(p=0.370)$.

At the time of the baseline examination, two patients were pain free (one patient in each of the groups with and without pseudo-disk). At the time of the follow-up evaluation, 23 patients were without pain, while 10 patients described pain. In the group without pseudo-disk, 12 patients (71\%) were pain free, and in the group with pseudo-disk, there were 11 (69\%).

Pain sensation on the VAS at the baseline evaluation was on average $56 \pm 38 \mathrm{~mm}$ (range $0-100$ ); $54 \pm 39 \mathrm{~mm}$ in the group without PD; and $59 \pm 39 \mathrm{~mm}$ in the group with PD. At the follow-up, a continued decrease could be seen: $22 \pm 36 \mathrm{~mm}$ (range 0-94); $21 \pm 37 \mathrm{~mm}$ in the group without PD; and $23 \pm 37 \mathrm{~mm}$ in that with PD. These improvements were statistically significant regardless of the presence of PD $(p<0.001)$ (Fig. 3).

In the baseline evaluation, 20 of the 33 TMJs showed a purely anterior displacement of the articular disk, one patient presented with an anterior-medial displacement and in 12 patients an anterior-lateral displacement was seen. At the follow-up evaluation, 16 of the 33 TMJs showed a purely anterior displacement of the articular disk, 5 TMJs an anterior-medial displacement, and 12 an anterior-lateral displacement. No significant difference in the distribution of disk position between patients with and without PD was seen in the baseline and the follow-up. Position and morphology of the condyle showed no statistical significant differences between the two groups. Disk shape at baseline evaluation was biconcave in 16 of the 33 TMJs evaluated, flat in 17 TMJs; no disk deformation or disk residues could be seen in any of the TMJs evaluated. Regarding disk shape at the time of the follow-up evaluation, it was biconcave in 15 of the 33 TMJs, flat in 15 and showed deformation in one TMJ of the group without PD; in two TMJs only disk residues could be seen. Neither the patients without $\mathrm{PD}$ nor those with $\mathrm{PD}$ showed any differences between baseline and follow-up evaluation. Twenty-five of the 33 TMJs showed homogenous disk signal intensity at the time of the baseline evaluation,
Fig. 1 a 28.12.2006: Parasagittal PD-sequence with open mouth in a patient with anterior disk dislocation without reposition and more severely impaired translation (condyle remains posterior to the articular eminence). b 14.10.2013: Parasagittal PD-sequence with open mouth 7 years later shows a faint pseudo-disk and range of mouth opening has slightly improved

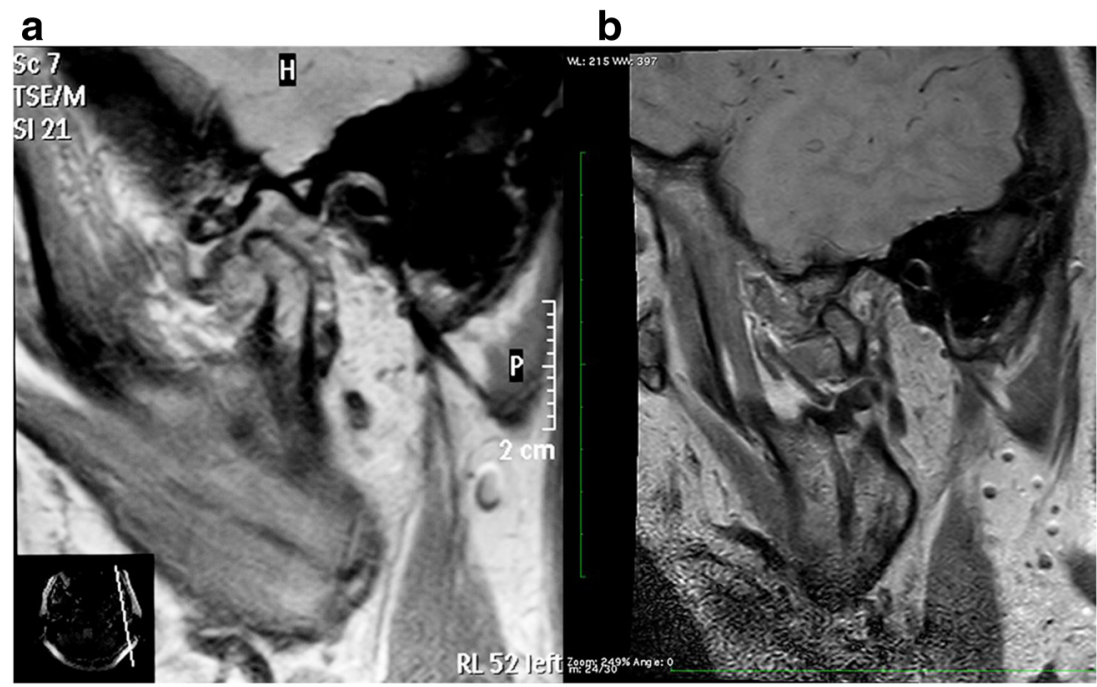


Fig. 2 a 24.7.2008: Parasagittal PD-sequence with open mouth in a patient with anterior disk dislocation without reposition $\mathbf{b}$ 3.11.2013: Parasagittal PDsequence with open mouth five years later without pseudodisk

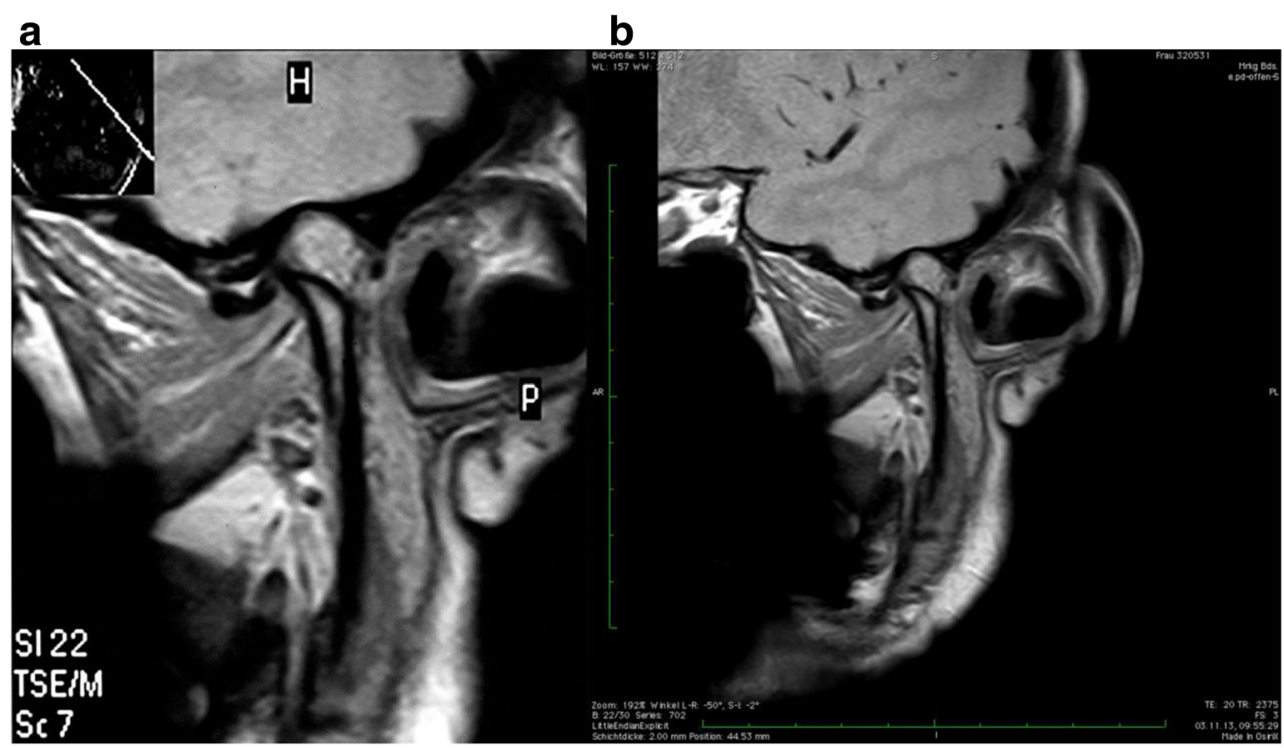

while eight disks showed focal hyperintensities. At the time of the follow-up evaluation, 23 of the 33 TMJs had homogenous disk signal intensity, while focal hyperintensities were seen in 10 disks. At the time of the baseline evaluation, 12 of the 33 TMJs showed a normal eminence, while 16 TMJs had a steep and five had a flat eminence. At the follow-up evaluation, 10 TMJs had a normal eminence, 13 had a steep and 10 had a flat eminence. No significant correlation was seen between the inclination of the eminence and the occurrence of a pseudo-disk. Of the 33 TMJs, four showed normal translational movement at the time of the baseline evaluation, while 28 TMJs showed restricted translational movement and one TMJ no translational movement at all. At the time of the follow-up evaluation, eight TMJs showed normal translational movement, 23 TMJs restricted translational movement and two no translational movement at all. The improvement of translation showed a statistical tendency in the group with PD $(p=0.064)$. There was no significant difference in translation between the two groups. At the time of the baseline evaluation, 17 TMJs had effusion, while no effusion was seen in 16 TMJs. At the follow-up evaluation, effusion was seen in 10 TMJs and no effusion was present in 23. A statistical tendency for a decrease of effusion was found in both groups ( $p=0.088$ group without $\mathrm{PD}, p=0.061$ group with PD), with no statistical significant difference between the two groups (Table 3).

Twenty-five patients ( $76 \%$ ) were treated with a combination of splint and physiotherapy, four patients (12\%) only received physiotherapy, two patients (6\%) only splint therapy, and two $(6 \%)$ no therapy at all.
The mean incisal edge distance before therapy was $41 \pm 8 \mathrm{~mm}$ (range 27-61 mm); $41 \pm 9 \mathrm{~mm}$ in the group without PD and $41 \pm 6 \mathrm{~mm}$ in the group with PD. A significant increase of incisal edge distance was seen in the majority of participants in the follow-up evaluation. The mean distance was $46 \pm 6 \mathrm{~mm}$ (range $33-60 \mathrm{~mm}$ ) with $45 \pm 7 \mathrm{~mm}$ in the group without and $46 \pm 5 \mathrm{~mm}$ in the group without PD.

The increase in mouth opening was statistically significant regardless of the presence of a PD $(p<0.001)$ (Fig. 4).

\section{Discussion}

This historical prospective investigation of morphological changes in the temporomandibular joint is a clinical

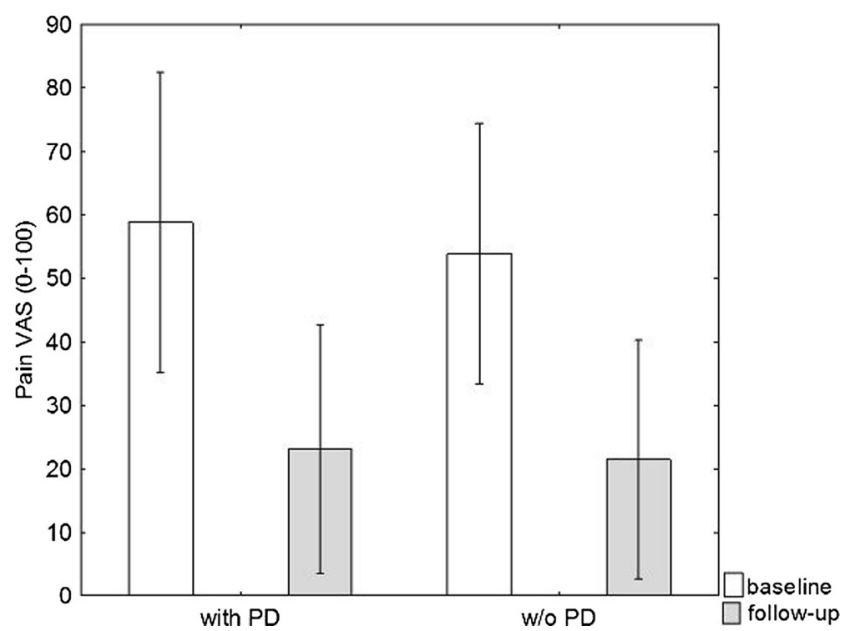

Fig. 3 Pain visual analog scale (VAS) at baseline and follow-up. Mean and $95 \%$ confidence intervals 


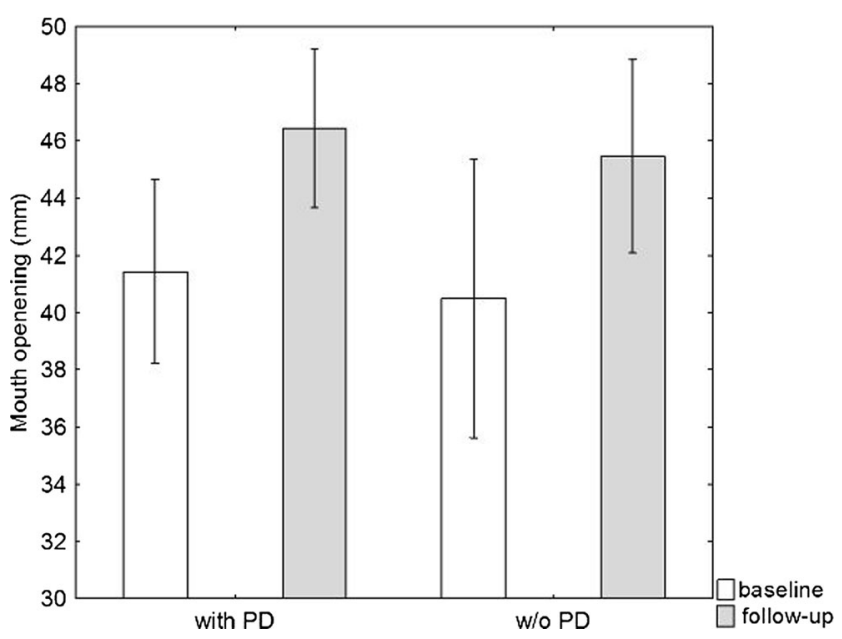

Fig. 4 Mouth opening (mm) at baseline and follow-up. Mean and $95 \%$ confidence intervals

and MRI study of patients with anterior articular disk displacement without reposition. Most of the patients were treated with physiotherapy and/or splint therapy. The group without therapy in the present study was too small for demonstrating statistically relevant results. The follow-up evaluation covered a time range of 4 8 years. At the end of this period, the patients underwent a second clinical functional analysis and MRI scans. In 42 TMJs, 19 pseudo-disks were evaluated corresponding to a rate of almost $50 \%$ with 17 of these pseudo-disks not seen in the baseline evaluation.

The use of higher field strengths in MRI and a dedicated coil has, compared to units with a field strength of 1.5 Tesla, allowed for improved imaging, especially for smaller joint structures $[5,6]$. The present study protocol optimizes delineation of condyle, disk, bilaminar zone, and disco temporal and disco condylar joint compartments and the surrounding tissue [9].

According to Bumann, progressive adaptation of the bilaminar zone can be demonstrated in 70-90\% of all TMJs with a varying degree of disk displacement. He notes that this can only be determined with certainty in the MRI by simultaneous demonstration of low-signal structures behind the disk in T1- and T2-weighted images [18].

A marked inclination of the articular eminence has been indicated as etiological factor favoring temporomandibular dysfunction $[19,20]$. As a result of the varying shape and inclination of the articular eminence, biomechanical components of the temporomandibular joint may change and thus play a key role in the development of an ADD [21]. The present study evaluated the inclination of the articular eminence, but no statistical significant correlation with pseudodisk formation or pain could be found.

Previous reports have compared the symptoms of treated patients with ADD without reposition versus untreated patients showing no significant differences $[12,13]$. In the present study, 25 patients $(76 \%)$ were treated with splintand physiotherapy, four patients $(12 \%)$ received only physiotherapy, two patients $(6 \%)$ were treated with splint therapy only, and two patients $(6 \%)$ were given no therapy at all. The fact that some patients do not require any additional therapy following detailed information is consistent with the results of Orthlieb and McNeill who postulated that rapid pain relief using medication, appropriate information on behavioral measures may be sufficient in some patients to achieve appropriate pain elimination and, thus, adequate treatment success $[22$, 23]. Our patients were also adequately instructed during the baseline evaluation to employ appropriate measures in the case of obvious parafunctional activity and to avoid any hard or chewy food until appropriate improvement of symptoms. This is in line with the findings of Sato who described marked improvement of symptoms or complete absence of pain in 21 of the pain patients in a "follow-up" study after 27.1 months, although they did not undergo any treatment. However, the anterior displacement of the disk persisted [24, 25]. Thus, mobility of the disk appears to be critical for successful pain therapy [26, 27]. In the present study in the group with pseudo-disk, more joints showed normal translation than in the baseline evaluation. Also in the group without pseudo-disk, the number of joints with no translation as well as with normal translation increased.

With a total number of 33 joints studied and with most patients receiving the combination therapy recommended in literature [28-30], no statistically significant correlation between formation of a pseudo-disk and pain relief could be demonstrated. At the follow-up evaluation, 21 patients showed an improvement of pain symptoms and 25 patients of mouth opening and even the two patients without therapy were free of symptoms after a period of about 12 months. These results were consistent with those reported by other authors [23, 31].

McNeill described adaptation of the splint after approximately 6 weeks during the initial phase as unavoidable in order to account for the ongoing neuromuscular changes of the maxillomandibular relationship and to improve temporomandibular disorders. This will be done until a stable, reproducible intercuspation position and adequate pain relief has been achieved [23]. In the present study, the splints were also adapted at regular intervals over a period of several weeks.

Ohnuki compared the effects of different therapeutic approaches (manual manipulation, arthrocentesis, and arthroscopic surgery) in patients with ADD without reposition and showed that — regardless of the type of treatment used - ADD without reposition persisted in $90 \%$ of the cases [26]. Similar results were reported after performing lavages in the TMJ, which were able to improve clinical symptoms, but could not correct the anterior disk displacement in most of the patients [24, 25, 32]. Even following arthroscopic surgery, disk position may frequently not be improved permanently $[26,27$, 


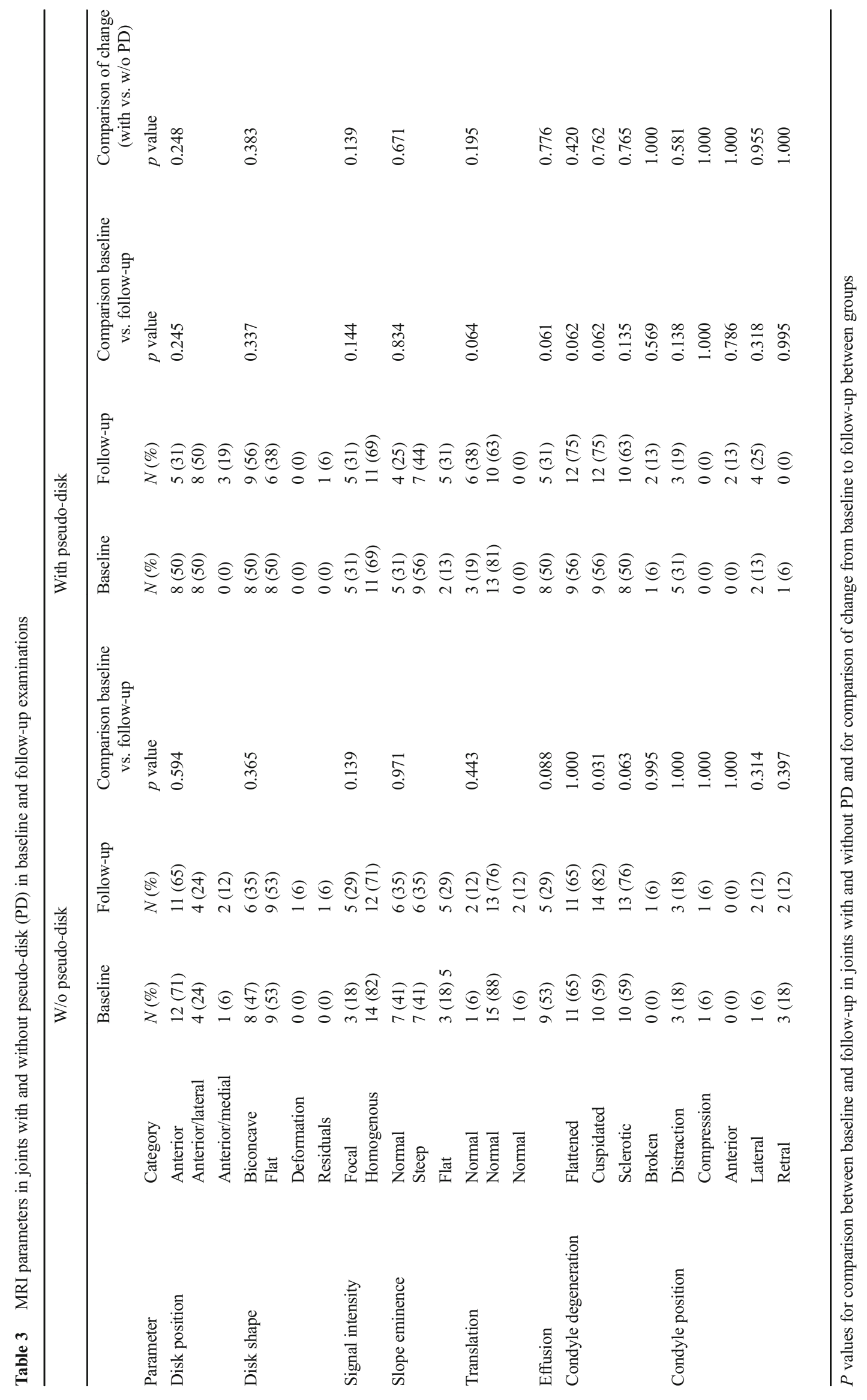


33]. A possible explanation for the general improvement of clinical symptoms may be the increased mobility of the articular disk following appropriate treatment [26]. Disk deformation in the TMJs treated with arthrocentesis and arthroscopic surgery was significantly higher than in patients treated with manual therapy or splint [26].

The particular strength of the present study includes the long time period of 4-8 years between the baseline and the follow-up evaluation of the patients as well as the exclusive use of the same dental and radiological center for all evaluations of the patients.

The number of study subjects must be considered as a shortcoming of the study, although for primary outcome variables of interest, pain and mouth opening, the study was sufficiently powered. As a result of the long time interval between the two evaluations, many patients eligible for inclusion could either not be contacted; on the other hand, only patients having undergone MRI scans with 3 Tesla at the baseline evaluation were enrolled in order to ensure comparability of the MRI images.

In summary, the present study could demonstrate a pseudodisk in almost half of the temporomandibular joints with an ADD without reposition. This is consistent with the results of previous studies describing morphological changes manifesting as fibrosis and neovascularization in the presence of chronic ADD without reposition in the retrodiscal region of the bilaminar zone.

Of the 31 patients describing pain on palpation in the temporomandibular joint and in the preauricular region upon mouth opening and at rest, 21 patients were pain free at the follow-up evaluation and in $80 \%$ of the patients significant improvement of mouth opening capacity could be achieved. Overall, the group with pseudo-disk showed similar results as the group without pseudo-disk. But there is some evidence that the improvement of the clinical and radiological parameters is more pronounced in the group with PD. Further prospective long-term studies should be performed to get more information about reparative processes of the temporomandibular joint with disk displacement without reposition.

Acknowledgments Open access funding provided by Medical University of Vienna.

Compliance with ethical standards The study was approved by the Ethics Committee of the Medical University of Vienna (1742/2012).

Conflict of interest All the authors declare that they have no conflict of interest in this study.

Funding This study was supported by the Department of Prosthodontics, University Clinic of Dentistry, Medical University, Vienna.

Informed consent Informed consent was obtained from all individual participants included in this study.
Open Access This article is distributed under the terms of the Creative Commons Attribution 4.0 International License (http:// creativecommons.org/licenses/by/4.0/), which permits unrestricted use, distribution, and reproduction in any medium, provided you give appropriate credit to the original author(s) and the source, provide a link to the Creative Commons license, and indicate if changes were made.

\section{References}

1. Stegenga B, De Bont LG, Boering G (1989) A proposed classification of temporomandibular disorders based on synovial joint pathology. Cranio 72:107-118

2. Kurita H, Kojima Y, Nakatsuka A, Koike T, Kobayashi H, Kurashina K (2004) Relationship between temporomandibular joint (TMJ)-related pain and morphological changes of the TMJ condyle in patients with temporomandibular disorders. Dentomaxillofac Radiol 335:329-333

3. Katzberg RW, Bessette RW, Tallents RH, Plewes DB, Manzione JV, Schenck JF, Foster TH, Hart HR (1986) Normal and abnormal temporomandibular joint: MR imaging with surface coil. Radiology 1581:183-189

4. Lemke AJ, Griethe M, Peroz I, Lange KP, Felix R (2005) Morphometric analysis of the temporomandibular joint with MRI in 320 joints. Rofo 1772:217-228

5. Schmid-Schwap M, Drahanowsky W, Bristela M, Kundi M, Piehslinger E, Robinson S (2009) Diagnosis of temporomandibular dysfunction syndrome-image quality at 1.5 and 3.0 Tesla magnetic resonance imaging. Eur Radiol 195:1239-1245

6. Stehling C, Vieth V, Bachmann R, Nassenstein I, Kugel H, Kooijman H, Heindel W, Fischbach R (2007) High-resolution magnetic resonance imaging of the temporomandibular joint: image quality at 1.5 and 3.0 Tesla in volunteers. Investig Radiol 426: 428-434

7. Katzberg RW, Tallents RH (2005) Normal and abnormal temporomandibular joint disc and posterior attachment as depicted by magnetic resonance imaging in symptomatic and asymptomatic subjects. J Oral Maxillofac Surg 638:1155-1161

8. Piehslinger E (2006) Grundlagen der zahnärztlichen Prothetik. 2. überarbeitete Auflage, Universimed Verlag Wien, Austria, p 163-164

9. Jäger L, Rammelsberg P, Reiser M (2001) Bildgebende Diagnostik der Normalanatomie des Temporomandibulargelenks. Der Radiologe 41:734-740

10. Isberg A, Westesson PL (1998) Steepness of articular eminence and movement of the condyle and disk in asymptomatic temporomandibular joints. Oral Surg Oral Med Oral Pathol Oral Radiol Endod 862:152-157

11. Farrar WB (1978) Characteristics of the condylar path in internal derangements of the TMJ. J Prosthet Dent 393:319-323

12. Lundh H, Westesson PL, Kopp S (1987) A three-year follow-up of patients with reciprocal temporomandibular joint clicking. Oral Surg Oral Med Oral Pathol 635:530-533

13. Westesson PL, Paesani D (1993) MR imaging of the TMJ. Decreased signal from the retrodiskal tissue. Oral Surg Oral Med Oral Pathol 765:631-635

14. Isberg A, Isacsson G, Johansson AS, Larson O (1986) Hyperplastic soft-tissue formation in the temporomandibular joint associated with internal derangement. A radiographic and histologic study. Oral Surg Oral Med Oral Pathol 611:32-38

15. Isberg A, Isacsson G (1986) Tissue reactions associated with internal derangement of the temporomandibular joint. A radiographic, cryomorphologic, and histologic study. Acta Odontol Scand 443: $160-164$ 
16. Kurita K, Westesson PL, Sternby NH, Eriksson L, Carlsson LE, Lundh H, Toremalm NG (1989) Histologic features of the temporomandibular joint disk and posterior disk attachment: comparison of symptom-free persons with normally positioned disks and patients with internal derangement. Oral Surg Oral Med Oral Pathol 676:635-643

17. Bjornland T, Refsum SB (1994) Histopathologic changes of the temporomandibular joint disk in patients with chronic arthritic disease. A comparison with internal derangement. Oral Surg Oral Med Oral Pathol 776:572-578

18. Bumann A, Lotzmann U (2000) Funktionsdiagnostik und Therapieprinzipien. In: Rateitschak KH, Wolf HF (eds) Farbatlanten der Zahnmedizin, 12. Georg Thieme Verlag Stuttgart, New York, p 171

19. Segami N, Suzuki T, Sato J, Miyamaru M, Nishimura M, Yoshimura H (2003) Does joint effusion on T2 magnetic resonance images reflect synovitis? Part 3. Comparison of histologic findings of arthroscopically obtained synovium in internal derangements of the temporomandibular joint. Oral Surg Oral Med Oral Pathol Oral Radiol Endod 956:761-766

20. Hall M (1985) Association between the prominence of the articular eminence and displaced TMJ disks. Journal of craniomandibular practice 33:238-239

21. Gökalp H (2001) Corelation between eminence steepness and condyle disc movements in temporomandibular joints with internal derangements on magnetic resonance imaging. Eur J Orthod 23:579-584

22. Orthlieb JD (2013) Propositions for a cognitive behavioral approach to bruxism management. J Stomat Occ Med 6:6-15

23. McNeill C, Danzig WM, Farrar WB, Gelb H, Lerman MD, Moffett BC, Pertes R, Solberg WK, Weinberg LA (1980) Position paper of the American Academy of Craniomandibular Disorders. Craniomandibular (TMJ) disorders-the state of the art. J Prosthet Dent 444:434-437

24. Sato S, Sakamoto M, Kawamura H, Motegi K (1999) Disc position and morphology in patients with nonreducing disc displacement treated by injection of sodium hyaluronate. Int J Oral Maxillofac Surg 284:253-257
25. Sato S, Sakamoto M, Kawamura H, Motegi K (1999) Long-term changes in clinical signs and symptoms and disc position and morphology in patients with nonreducing disc displacement in the temporomandibular joint. J Oral Maxillofac Surg 571:23-29 discussion 29-30

26. Ohnuki T, Fukuda M, Nakata A, Nagai H, Takahashi T, Sasano T, Miyamoto Y (2006) Evaluation of the position, mobility, and morphology of the disc by MRI before and after four different treatments for temporomandibular joint disorders. Dentomaxillofac Radiol 352:103-109

27. Moses JJ, Sartoris D, Glass R, Tanaka T, Poker I (1989) The effect of arthroscopic surgical lysis and lavage of the superior joint space on TMJ disc position and mobility. J Oral Maxillofac Surg 477: 674-678

28. Truelove E, Huggins KH, Mancl L, Dworkin SF (2006) The efficacy of traditional, low-cost and nonsplint therapies for temporomandibular disorder: a randomized controlled trial. J Am Dent Assoc 1378:1099-1107 quiz 1169

29. Lundh H, Westesson PL, Eriksson L, Brooks SL (1992) Temporomandibular joint disk displacement without reduction. Treatment with flat occlusal splint versus no treatment. Oral Surg Oral Med Oral Pathol 736:655-658

30. Wright EF, Schiffman EL (1995) Treatment alternatives for patients with masticatory myofascial pain. J Am Dent Assoc 1267:1030 1039

31. Peroz I (2004) Untersuchungen zur Discusverlagerung ohne Reposition am Kiefergelenk. Habilitation aus dem Zentrum für Zahnmedizin der Medizinischen Fakultät Charité der Humboldt Universität zu Berlin

32. Emshoff R, Rudisch A (2003) Are internal derangement and osteoarthrosis linked to changes in clinical outcome measures of arthrocentesis of the temporomandibular joint? J Oral Maxillofac Surg 6110:1162-1167 discussion 1167-70

33. Montgomery MT, Van Sickels JE, Harms SE, Thrash WJ (1989) Arthroscopic TMJ surgery: effects on signs, symptoms, and disc position. J Oral Maxillofac Surg 4712: $1263-1271$ 